International Journal of Advanced Statistics and Probability, $8(1)(2020) 5-9$
International Journal of Advanced Statistics and Probability
Website: $w$ ww.sciencepubco.com/index.php/IJASP
Research paper

\title{
Network analysis of countries' partnership in European sports programs: Erasmus+ sport
}

\author{
Ioannis Dallas ${ }^{1}$, Ioannis Ntoumanis ${ }^{2}$, Francesca Karatza ${ }^{1}$, Georgios Ch. Makris ${ }^{1}$ * \\ ${ }^{1}$ Inter-Faculty Master Program on Networks and Complexity, Aristotle University of Thessaloniki \\ ${ }^{2}$ Department of Mathematics, Aristotle University of Thessaloniki \\ *Corresponding author E-mail: geormak@gmail.com
}

\begin{abstract}
In the present work, data analysis of Erasmus+ Sport programs was performed using Network Theory. Funding amounts and partner countries per program are the information of the target data. Developing a Python-based program, a network of countries' partnerships has been developed to examine whether specific countries cooperate more frequently, and which countries participate in more Erasmus+ Sport programs. Thus, some basic indicators of centrality from network theory were calculated, which are presented together with their mathematical interpretation.

It has also been studied whether the number of a country's participation in these programs is affected by its economic or social national characteristics. Specifically, GDP, happiness and education indexes are all examined if they affect a country's participation. Finally, given how the funding amount of a program is split between the partner countries, the total amount of funding received by each country for the period 2014-2018 was calculated.
\end{abstract}

Keywords: Cooperation; Erasmus+; Indexes; Networks; Sports.

\section{Introduction}

The data comes from the 'Partnerships' program, which is provided free of charge by Erasmus+ and is available on the program's official website [1], [2]. They describe the structure of the programs and provide details on each of the 600 programs carried out in.

\section{Networks construction}

A network of partnerships (unweighted) has been created in which countries that have participated in at least one research program are represented as nodes and their involvement in a program indicates the link between them. Then, the network of partnerships was converted to weighted [3] and the repetition of the two countries' cooperation in different programs was set as weight $(+1)$

\section{Centralization measures and network properties}

\subsection{The paper should have the following structure}

For the weight-free network (simple cooperation of two countries in one program), relatively high values of centrality appear in several countries. Some of them are IT, UK, FR, BE, DE. While less central are: GE, AL, BR, IN, MY and MC.

For the weighted network (multiple cooperation of two countries in different programs), a clear picture is given of the countries that cooperate more dynamically in different programs

Italy has a dominant role, followed by a network of partnerships: UK, EL, ES, FR, PT, BG. The less central countries appear to coincide with the previous figure, which is to be expected, as their weak co-operation in one program renders to a weak co-operation in other programs as well. Moreover, this difference is also confirmed by the graphical representation of the two centrality indices [4], [5]. 

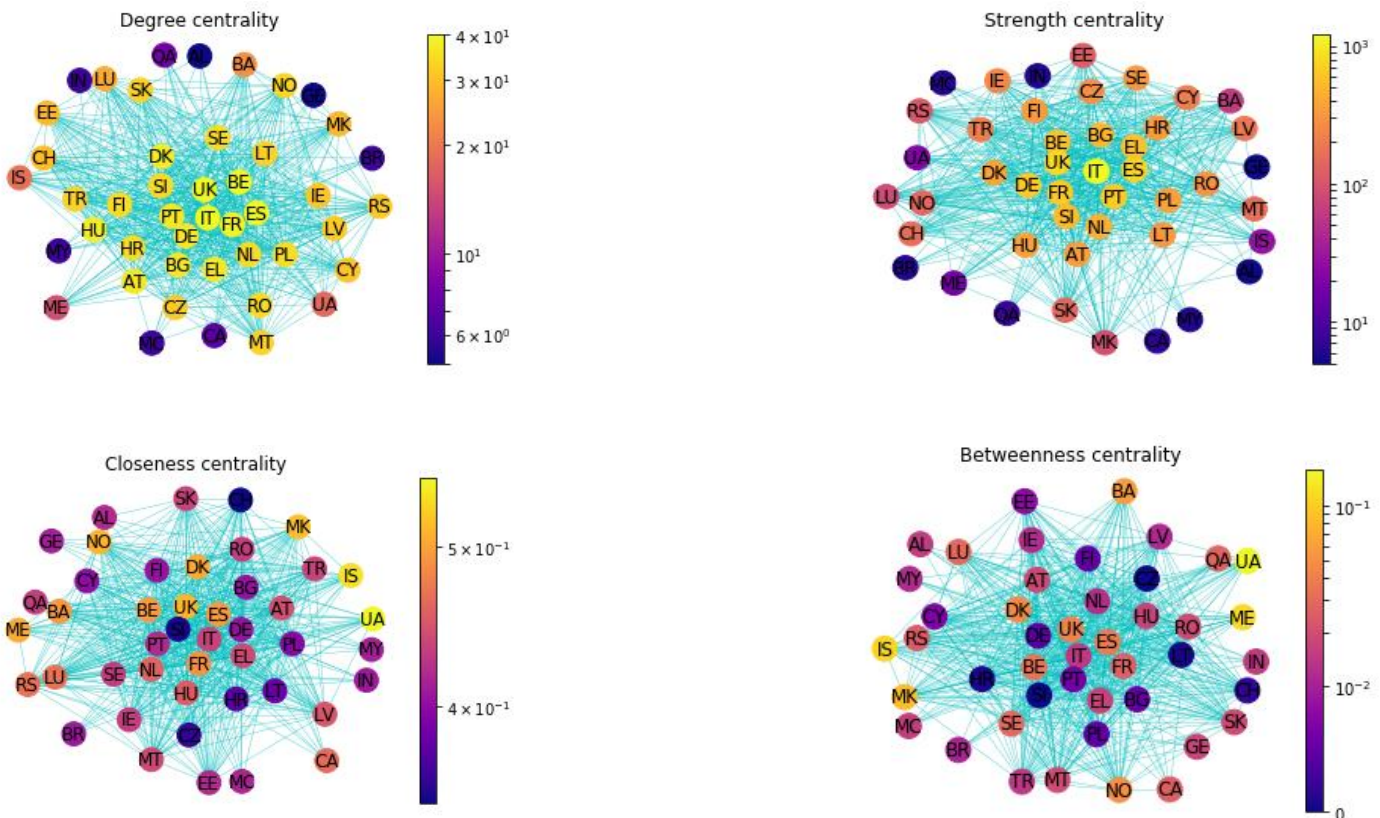

The diagrams above illustrate that UA, IS, ME, MK nodes are the most frequently encountered in establishing cooperation between two countries in different programs.
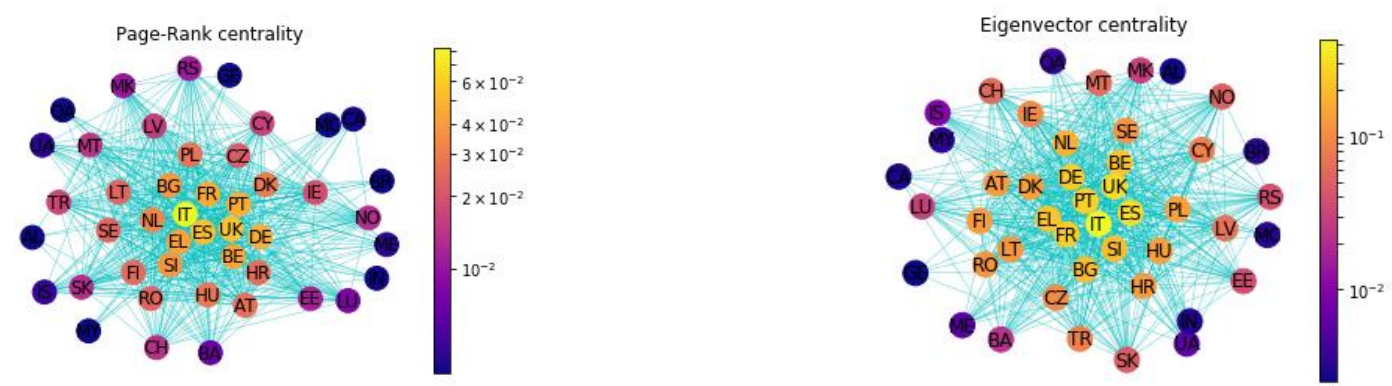

Since the network is unweighted, the concept of 'better' in-degree [6] is typically considered indifferent However, this is not a problem for the measurements of the above centrality measures. The Page-Rank algorithm has the advantage of taking into account the weight, thereby enabling it to give a clearer picture of the countries linked to different programs. Therefore, it appears that Italy is in a dominant position.
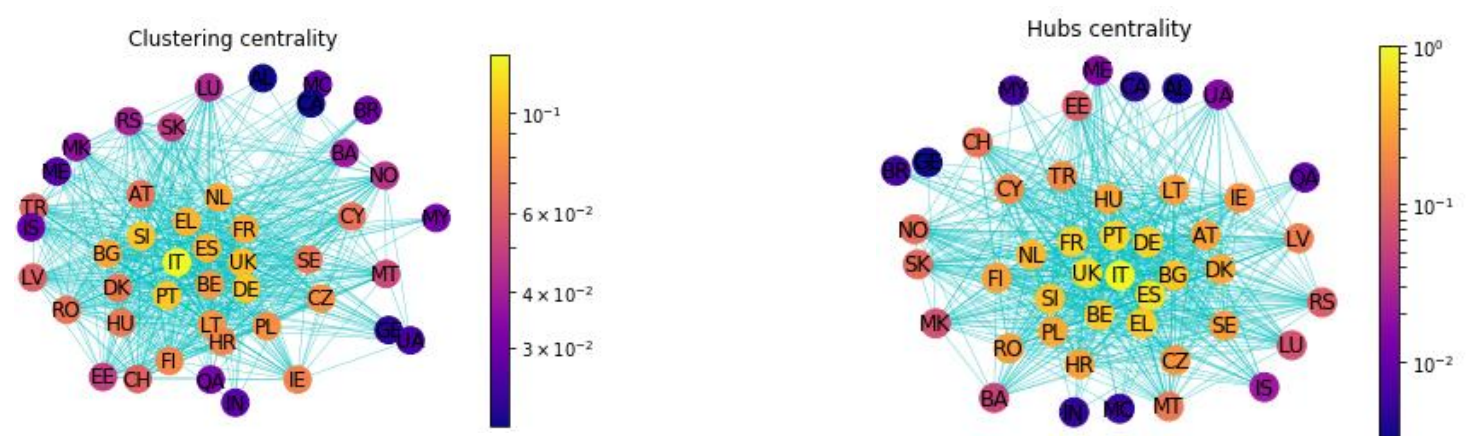

From the clustering index [7], [8], [9] it is noted that Italy and the DE, UK, PT, SI, BG, EL, NL, ES clusters are the most involved nodes in generating different clusters. This fact is combined with the Hubs index and essentially highlights those nodes that are more generally involved in creating collaboration through different programs. The highest nodes are IT, UK, PT, FR, ES, DE, EL.

\subsection{The paper should have the following structure}

In some cases, dependence has been observed between centrality indices in a network [10], [11], [12]. Below are the answers.

The graph seems to follow a polynomial degree function. What is observed is that the strength centrality values rise rapidly for small changes in degree centrality values. In practice, this means that the number of programs involved in one country with another (weight) grows more than the simple link between them in one program. 

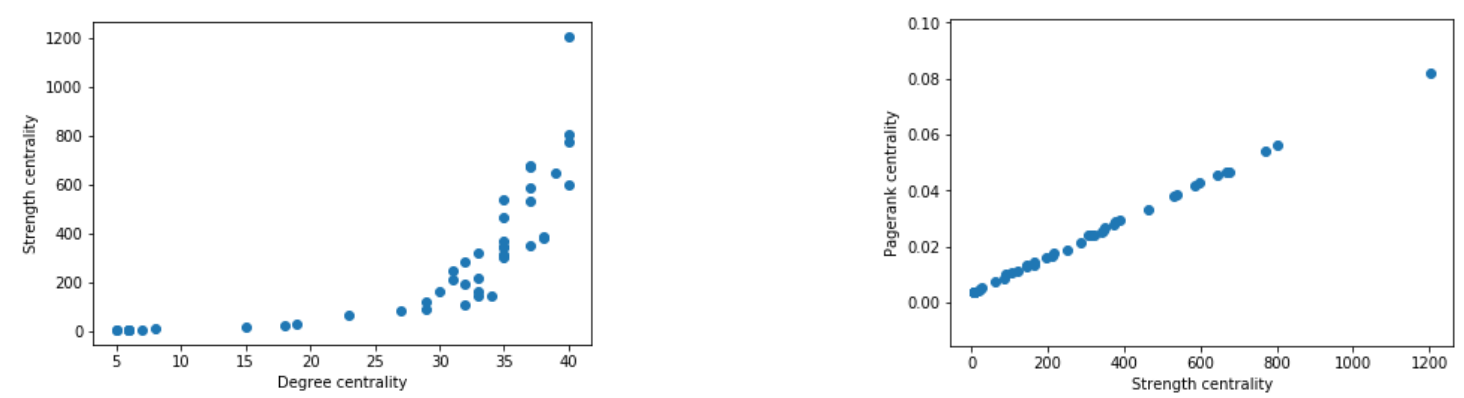

It is noted that Eigenvector, PageRank [8], and hub centrality [8] formed graphs similar to the graph above. This is explained by the theory of networks, as well as the example of its application in the connection of sports programs between countries. In essence, the three centralities are a kind of generalization of the gradual centrality [10], [11], and deeper interpretation of the network. They aim to identify the most important countries that provide the most information through other countries. In particular, with the hub centralization being a generalization of self-centeredness, the countries involved are more clearly identified and therefore may be coordinating some programs

In particular, it appears that PageRank's centrality values increase concerning centrality strength values (linearly dependent). In practice, this means for the network of countries that as the number of cooperating programs increases, some countries play a more important role in working together. The same was true for the Hubs - Strength, and Eigenvector - Strength graphs.
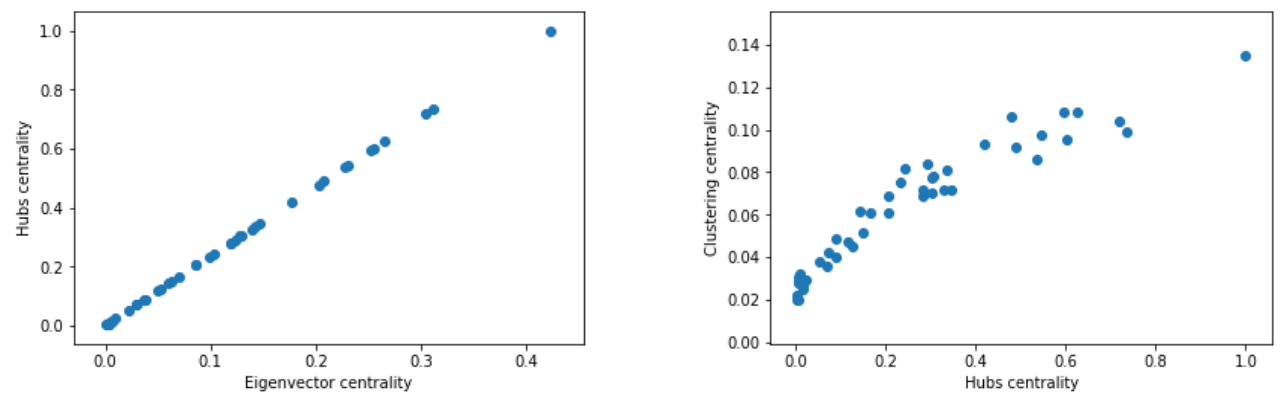

The first figure indicates that there is a strong linear relationship between the hub centrality and the eigenvector centrality. Given that the former is a generalization of the latter, such a relationship is reasonable. What is again highlighted is the weight increase rate of the countries which participate to the programmes the most frequently.

The linear relationship between the hub centrality and the clustering coefficient is also strong, according to the second figure. That means that the more frequently a country participates in Eramsus Sports programmes (hub), the more frequently its graph neighbours collaborate in such programmes. This indicates that indirect connections between specific countries are vulnerable, in case another country attempts to collaborate with them.

It is also worth mentioning that in both figures there is a single point that is relatively isolated. This indicates that the network includes a leading country, which collaborates with many others and therefore has high centrality indices.

\subsection{Correlation between financial/social indices and centralities}

In this paragraph, it is examined whether strength centrality is correlated with social and financial indices, such as GDP, happiness index and education index. Such an approach might be interested because: If, for instance, strength is positively correlated with GDP, that would mean that the highest the GDP of a country, the more frequently that country participates in Erasmus Sports programmes.

Gross Domestic Product (GDP) is the monetary value of all finished goods and services made within a country during a specific period, often annually. According to World Development Indicators data [13], the average GDP for the period 2014-2018 was calculated for each country, since this is the graph's period of interest. The following first figure illustrates the relationship between the resulting average GDP with the strength centrality.
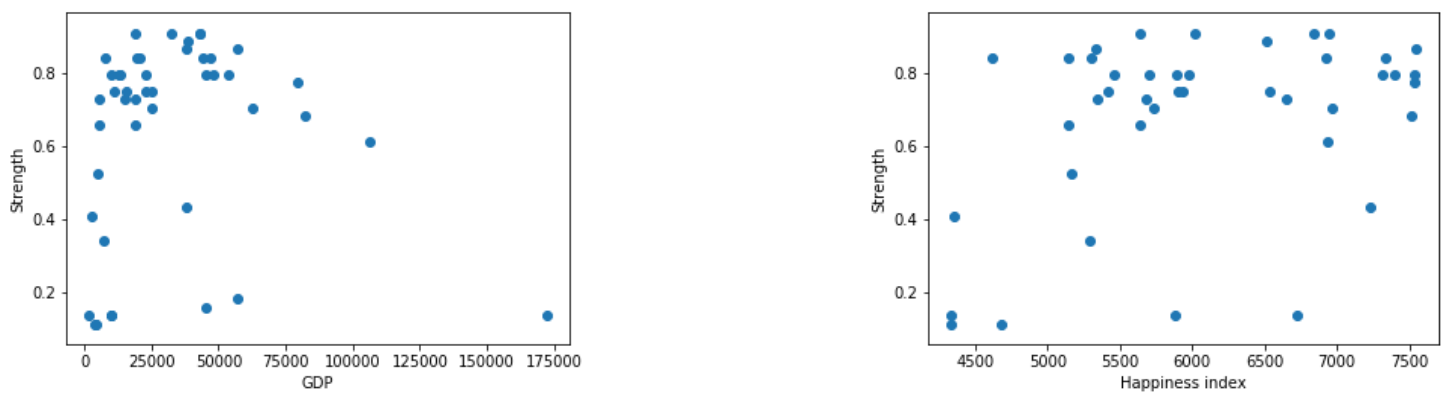

GDP is relatively low for most of the countries the data contain, whereas the strength is more widely distributed. In addition, Pearson's correlation coefficient is -0.013 and therefore, the two variables are not correlated.

United Nations (UN) have defined an index that measures each country's citizens' happiness, the happiness index. This index takes into consideration: GDP, social support, expected lifetime, personal freedom, charity and corruption. The average happiness index was calculated in accordance with the average GDP, for the period 2014-2018. The second figure illustrates the relationship between the resulting 
average happiness index with the strength centrality. Pearson's correlation coefficient is 0.3887 and therefore, the two variables are weakly correlated.

The same procedure was followed for the education index. Education index is part of the Human Development Index that UN publishes annually, and is calculated by taking into account the average length of education (in years) and the expected education length for each country. The average happiness index was calculated in accordance with the average GDP, for the period 2014-2018. The following figure illustrates the relationship between the resulting average education index with the strength centrality. Pearson's correlation coefficient is 0.56 and therefore, the two variables are weakly correlated.

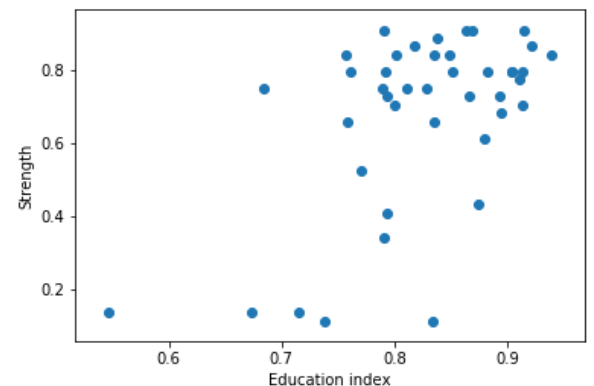

The relationships discussed in this paragraph are summarized in the next table.

\begin{tabular}{llll}
\hline 3 & GDP & Happiness index & Education index \\
\hline Strength & -0.0134 & 0.3887 & 0.5615 \\
\hline
\end{tabular}

The above values are relatively low and therefore, no strong relation seems to exist. So, national indices, such as GDP, happiness index and education index are not correlated with the number of times a country participates in Eramsus Sports programmes.

\subsection{Grants per country}

All Erasmus+ Sports programmes are based on grants, which is shared between the participating countries. In fact, all countries but the Coordinator take the same percentage, whereas the Coordinator takes the double. If, for instance, 4 countries participate in a programme, then the Coordinator will gain $2 / 5$ of the total grant, whereas the remaining 3 countries will gain 1/5 each.

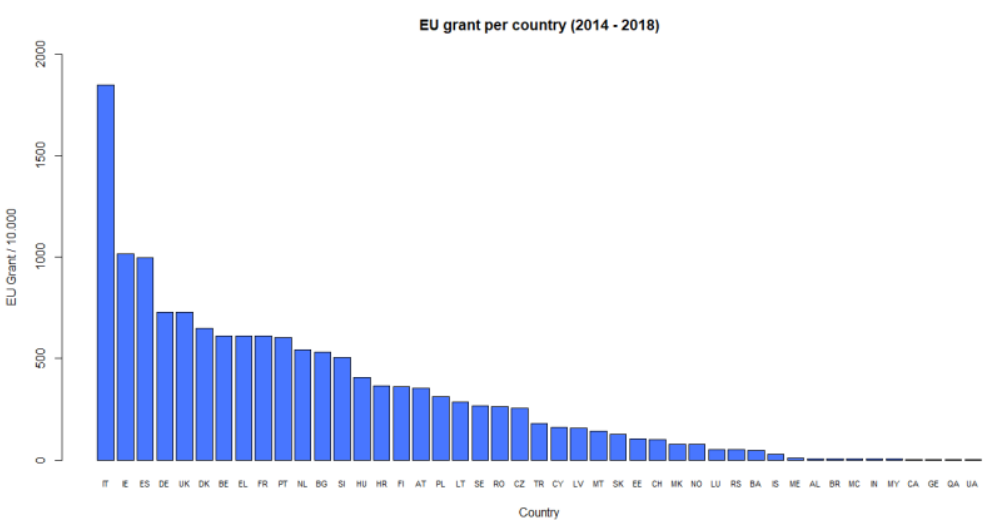

As the above figure illustrates, the grants are not uniformly distributed, which means that certain countries receive more/higher grants than others. Particularly, Italy seems to be the leading country in terms of the receiving grants. Ireland, Spain, Germany and UK also receive high grants. Countries outside the European Union, such as Qatar and Georgia, receive the lowest grants, since they can only participate in certain programmes and under certain circumstances. Greece comes 8 th in the above scale.

\section{Conclusion}

GDP, happiness index and education index are not correlated with the strength centrality, that is the total participation in Erasmus+ Sports programs.

Italy is the country that participates the most frequently in Erasmus+ Sports programs. This fact was highlighted by calculating several centralities, as well as by calculating the amount of grants it receives.

Certain countries, such as Germany, England, Bulgaria, Holland and Slovenia, seem to form a clique in the network. However, this cliqueform is quite vulnerable due to low values of clustering coefficient.

Mediterranean countries have relatively high centrality values, which indicates that Mediterranean has a leading role in forwarding, advertising and supporting athletic standards.

In addition, bridge-countries are detected, which perform as intermediate nodes when other countries connect. In fact, Ukraine, Iceland, Montenegro and North Macedonia perform as bridge-countries.

Grants and strength were relatively correlated, but Ireland is an exemption. Despite the high grants it receives, its role in the network is not important (low centrality values). This might be due to participation in many different programmes as Coordinator (double grant).

What has been previously studied is collaboration between players in diverse sports, as well as statistical differences between teams [14]. Therefore, the collaboration between countries in the Erasmus+ Sport network highlights the strategic importance of supporting sports.In this section you should present the conclusion of the paper. Conclusions must focus on the novelty and exceptional results you acquired. 
Allow a sufficient space in the article for conclusions. Do not repeat the contents of Introduction or the Abstract. Focus on the essential things of your article.

\section{References}

[1] https://ec.europa.eu/programmes/erasmus-plus/about_el.

[2] https://eacea.ec.europa.eu/erasmus-plus/actions/sport_en.

[3] Cohen, A. M. (1964). "Communication networks in research and training". Personnel 128 Administration. 27, 18-24.

[4] Rogers, D. L. (1974). Sociometric analysis of interorganizational relations: application of theory and measurement. Rural Sociology. 39(4), 487-503.

[5] Freeman L. C. (1977). A set of measures of centrality based on betweenness. Sociometry Vol. 40(1), 35-41 https://doi.org/10.2307/3033543.

[6] Freeman, L. C. (1979). Centrality in social networks conceptual clarification. Social networks. 1(3), 215-239. https://doi.org/10.1016/03788733(78)90021-7.

[7] Everett, M. G., \& Borgatti, S. P. (1999). The centrality of groups and classes. Journal of Mathematical Sociology. 23(3),181-201. https://doi.org/10.1080/0022250X.1999.9990219.

[8] Grolmusz, V., (2012). A note on the pagerank of undirected graphs, [Online]. Available: http://arxiv.org/abs/1205.1960.

[9] Newman, M. (2010). Networks: An Introduction. Oxford University Press Inc., New York.

[10] Watts, D. J., \& Strogatz, S. H. (1998). Collective dynamics of 'small-world'networks. Nature. 393(6684), 440-442. https://doi.org/10.1038/30918.

[11] Körner, János (1973). "Coding of an information source having ambiguous alphabet and the entropy of graphs". 6th Prague conference on information theory: $411-425$.

[12] Seyed Saeed Changiz Rezaei, Chris D. Godsil:

[13] Entropy of symmetric graphs. Discrete Mathematics 339(2): 475-483 (2016). https://doi.org/10.1016/j.disc.2015.09.020.

[14] https://databank.worldbank.org/source/world-development-indicators.

[15] Clemente, F.M. et al. (2016). Social Network Analysis Applied to Team Sports Analysis, Springer Briefs in Applied Sciences and Technology https://doi.org/10.1007/978-3-319-25855-3. 